\title{
Spatiotemporal Regularity and Interevent Contingencies as Information for Infants' Visual Expectations
}

\author{
Naomi Wentworth \\ Department of Psychology \\ Lake Forest College \\ Marshall M. Haith and Roberta Hood \\ Department of Psychology \\ University of Denver
}

This study examined infants' use of picture-location contingencies and spatiotemporal regularity in forming visual expectations. Ninety-six 3-month-olds watched an event sequence in which pictures appeared at 3 locations, either in regular leftcenter-right alternation or in a random center-side pattern. For half of the infants, the content of the central picture was predictive of the location of the upcoming peripheral event. Analyses of anticipations and interpicture fixation shifts revealed that both spatiotemporal regularity and consistent interevent contingencies fostered increased anticipation of peripheral pictures. The type of spatiotemporal sequence that infants observed also differentially biased their responses to test trials that followed the picture sequence: Infants who experienced regular alternation sequences continued the side-to-side pattern during the 2-choice test trials, whereas infants who experienced irregular sequences looked back to the location of the previous picture. Stable interevent contingencies, in contrast, did not bias infants' responses toward the contingent side during the choice test trials.

Studies by Haith and his collaborators (e.g., Haith, Hazan, \& Goodman, 1988; Haith, Wentworth, \& Canfield, 1993) have shown that by the age of 2 or 3 months, infants can form expectations about the locations of upcoming events

\footnotetext{
Requests for reprints should be sent to Naomi Wentworth, Department of Psychology, Lake Forest College, 555 North Sheridan Road, Lake Forest, IL 60045. E-mail: wentworth@1fc.edu
} 
in a regular sequence of brief picture presentations. Infants then use their expectations either to make anticipatory eye movements to the sites of upcoming pictures or to react more quickly after the pictures appear. This capacity of the young infant to form expectations for the locations of upcoming pictures is impressive because it involves several visual information-processing skills. That is, infants must detect the spatiotemporal regularity that governs the sequence of picture presentations, form expectations for what comes next, and then act on those expectations. In addition to detecting where and when pictures are presented, infants also pay attention to the content of the pictures (i.e., what is shown), as indicated by the finding that stable picture content facilitates the formation of expectations about where and when pictures will appear (Wentworth \& Haith, 1992).

Although 2- and 3-month-old infants can use spatiotemporal regularity and stable picture content to form expectations about upcoming events, a study by Johnson, Posner, and Rothbart (1991) suggests that reliable interevent contingencies may not be as informative for infants until somewhat later in life. In the Johnson et al. study, 2-, 3-, and 4-month-olds were given 18 training trials during which a central stimulus was followed by a peripheral target after a $1,000-\mathrm{msec}$ delay. Two central stimuli were used: One preceded peripheral targets on the left and the other preceded peripheral targets on the right. Following a short break, an 18-trial test session was completed with three types of test trials presented in a random order. On contingency test trials, one of the central stimuli was presented and, following the standard 1,000-msec delay, both peripheral targets appeared simultaneously. Only the 4-month-olds showed a significant bias in looking toward the side that had been associated with the central stimulus during the training session.

It is possible that infants can use spatiotemporal regularity as information for visual expectations before they can use interevent contingencies. If so, at 2 months of age, infants would be able to form expectations about upcoming events in a regular sequence of alternating pictures, as in the Haith et al. (1988; Haith et al., 1993) studies, but they would be unable to use a contingent relation between stimuli to predict the location of an upcoming stimulus, as in the Johnson et al. (1991) study. The extensive division of labor that has been found within the visual system (e.g., Kaas, 1989; Zeki, 1993), both for parsing incoming visual information and for executing eye movements, suggests that various discontinuities in the development of visual information processing might be expected. In fact, Johnson (1990) suggested that differential development in four pathways that control eye movements can account for several age differences in early visual behavior. Although Johnson's model does not distinguish among anticipatory saccades that are made in response to different types of visual input, such as spatiotemporal regularity or interevent contingencies, it could easily be adjusted to do so. 
Alternatively, it is possible that the apparent developmental discontinuity in the types of information that infants can use to form visual expectations is an artifact of the different paradigms that have been used. That is, in the visual expectation paradigm (VExP), used to study the development of spatiotemporal expectations, infants typically watch 60 pictures that are presented in regular alternation about $6^{\circ}$ to the left and right of center on a video monitor. In contrast, in the study of interevent contingencies by Johnson et al. (1991), infants saw only 18 training trials, with targets presented at fairly large eccentricities ( $34^{\circ}$ to the left and right). Moreover, the two central stimuli were more salient $\left(5^{\circ}\right.$ shapes that either moved or loomed while bleeping) than the single peripheral target (a $3^{\circ}$ flashing green diamond above a pink rectangle). Thus, with fewer training trials, larger stimulus eccentricities, and less salient peripheral targets, the paradigm Johnson et al. used may have underestimated young infants' ability to use interevent contingency information. One goal of this study was to use a single paradigm to determine whether young infants can form visual expectations on the basis of both interevent contingencies and spatiotemporal regularity. To this end, the VExP was modified so that pictures appeared at three locations: at the center $(\mathrm{C})$ and at $10^{\circ}$ to the left (L) and right (R) of center. For half of the infants, there was a contingent relation between the content of the central picture and the location of the subsequent peripheral picture. If interevent contingency information contributes to their expectations, infants should use the identity of the central stimulus to anticipate peripheral pictures during the course of the VExP sequence, and they should look to the "correct" side during contingency test trials at the end of the 60-picture sequence.

A second purpose of the study was to examine the relation between the ability to make anticipatory eye movements and the ability to disengage attention from a visual target. Several investigators (e.g., Atkinson, Hood, Braddick, \& WattamBell, 1988; Atkinson, Hood, Wattam-Bell, \& Braddick, 1992; Frick, Colombo, \& Saxon, 1999; Hood \& Atkinson, 1993; Johnson et al., 1991; Reznick, 1994) have noted that young infants make saccades to a peripheral target less readily while another fixation stimulus is available. According to Johnson's (1990) model, maturation of an inhibitory pathway from the visual cortex to the superior colliculus, via the substantia nigra at about 1 month of age, is responsible for the appearance of this relative difficulty in disengaging attention from a target under scrutiny. As additional pathways mature, for example, from the visual cortex to the frontal eye fields (Johnson, 1990) or from the posterior parietal lobe to elsewhere in the eye movement system (e.g., Fisher \& Breitmeyer, 1987; Hood, 1995), infants are better able to look away from a central picture to inspect a target in the periphery. Because these pathways also play a role in the execution of voluntary shifts in visual fixation, their maturation is considered crucial for the development of anticipatory saccades. As a consequence, infants who experience difficulty in turning away from a central stimulus to look at a peripheral target should be less likely to 
anticipate upcoming pictures and, conversely, infants who are more likely to make anticipatory saccades should be able to turn away from a central stimulus to inspect a peripheral target. To test this prediction, this study included four disengage attention test trials on which the central picture remained visible while a peripheral picture was presented, and performance was correlated among VExP and attention disengagement tasks.

Finally, this study examined the interaction between the visual search strategies that infants initially bring to the visual expectation task and the demands of the VExP. In a previous study, Wentworth and Haith (1998) found that 2- and 3-month-old infants began the VExP session with a bias to make eye movements during the intervals between picture presentations that were consistent with the direction of their fixation shifts to the prior picture. Given the spatial alternation of the VExP, infants must have overcome this initial repetitive response bias to make (alternating) anticipatory eye movements to the locations of upcoming pictures. It is possible that infants would be better able to anticipate upcoming pictures if doing so did not require them to overcome their initial tendency to make repetitive saccades. Modification of the VExP to include three picture locations allowed us to address this possibility. During regular side-to-side alternation among the three locations, infants could anticipate peripheral pictures by repeating the direction of their previous saccade, but they could anticipate the center stimulus only by making a saccade in the opposite direction. To illustrate, consider a segment of the sequence in which pictures appeared successively at the L, C, and R locations. After having viewed the first picture, at $\mathrm{L}$, the infant could make a saccade to the right to view the second picture, at $\mathrm{C}$. Then, to examine the next picture, at $\mathrm{R}$, the infant would again make a saccade to the right, repeating the direction of the previous saccade. To return to the center from the $\mathrm{R}$ location, however, the infant would have to make an opposite-direction, leftward, saccade. Thus, if infants have a bias to repeat the direction of their saccades, they should anticipate more peripheral than central pictures during regular side-to-side alternation sequences.

\section{METHOD}

\section{Sample}

Ninety-six 3-month-old infants (83-96 days, $M=89.7, S D=3.08$ ) were randomly and equally assigned to one of four groups that differed in whether or not the spatiotemporal sequence they watched was regular and in whether or not a contingent relation existed between the central stimulus and the location of the next peripheral picture. Infants were selected from a pool of healthy, full-term infants born to predominantly middle-class families in the Denver metropolitan area. Parents were given a certificate of participation for their infant and $\$ 5$ to help 
defray the cost of transportation to the laboratory. An additional 31 infants were brought to the laboratory but not included in the sample. Of these 31 infants, 27 were excluded because they looked at fewer than 45 pictures during the VExP session, 3 were excluded because of equipment failure, and 1 was excluded because of experimenter error.

\section{Stimuli}

Computer-generated pictures, approximately $4^{\circ}$ square, appeared alternately at the $\mathrm{C}$ of a video monitor and at one of the two peripheral locations $10^{\circ}$ to the $\mathrm{L}$ or R of center. Either of two pictures could appear at the central location: One was a three-dimensional cube that rotated once per $700 \mathrm{msec}$; the other was a circular wheel composed of four colors that spun $360^{\circ}$ within the circular frame, once per $700 \mathrm{msec}$. One of eight different pictures appeared at one of the two peripheral locations. Peripheral pictures were colorful, dynamic shapes such as spinning arrows, looming and receding squares, schematic faces, and flashing stars.

\section{Apparatus and Procedure}

Pictures were displayed on a video monitor that the infant could see by reflection on an overhead mirror. A collimated light source, with filters to reduce heat and visibility, illuminated the infant's right eye to enable infrared corneal reflection photography for monitoring eye movements. A video camera, sensitive to light in the infrared range, recorded the infant's right eye during the session (see Haith et al., 1988, for details of apparatus and recording procedure). To facilitate later coding of eye movements, the time, date, and stopwatch video output from a time-date generator were superimposed on each field of the videotaped eye image; one digit of the time-date display was modified to indicate picture onset and offset.

While watching the pictures, infants lay supine on a mattress with their heads resting in a cloth sling to restrain head movement. Before the session began, a pacifier was offered to the infant, the infant's right eye was positioned in the center of the camera's range of view, the camera was focused, and the room lights were extinguished. Each infant participated in three tasks. For the first task, infants watched one of four 60-picture VExP sequences, with each sequence consisting of pictures alternating between a central location and peripheral locations $10^{\circ}$ to the left or right of center. Two sequences consisted of regular side-center appearances, whereas the other two sequences consisted of irregular center-side appearances. In addition, for two sequences there was a contingent relation between the content of the central picture and the location of the following peripheral 
picture, and for the other two sequences there was not a contingent center-side relation. Sequence 1 (regular + contingent) followed a regular spatiotemporal pattern ( $\mathrm{L}, \mathrm{C}, \mathrm{R}, \mathrm{C} \ldots$ ) with a contingent relation between the central stimulus and the location of the subsequent peripheral picture (i.e., $C_{1}$ preceded an $L$ picture and $C_{2}$ preceded an $\mathrm{R}$ picture, counterbalanced over infants). Sequence 2 (irregular + contingent) followed an irregular spatiotemporal pattern ( $\mathrm{L}, \mathrm{C}, \mathrm{R}, \mathrm{C}, \mathrm{R}, \mathrm{C}, \mathrm{L} \ldots$... ) with a contingent relation between the central stimulus and the location of the subsequent peripheral picture. Sequence 3 (regular + not contingent) followed a regular spatiotemporal pattern with no contingent relation between the central stimulus and the subsequent peripheral picture (i.e., $\mathrm{C}_{1}$ equally preceded $\mathrm{L}$ and $\mathrm{R}$ pictures). Sequence 4 (irregular + not contingent) followed an irregular spatiotemporal pattern with no contingent relation between the central stimulus and the location of the subsequent picture. In all cases, picture duration was $700 \mathrm{msec}$ and the interpicture interval was $1,000 \mathrm{msec}$. Each sequence consisted of 30 picture presentations at the central location and 15 presentations at each of the two peripheral locations ( $\mathrm{L}$ and $\mathrm{R}$ ).

Immediately after the 60-picture VExP sequence, infants received a set of four contingency test trials to assess their use of the contingent relation between the central stimulus and the subsequent picture. Contingency test trials consisted of presentation of a central stimulus $\left(\mathrm{C}_{1}\right.$ or $\left.\mathrm{C}_{2}\right)$ for $700 \mathrm{msec}$ followed by the standard interpicture interval of $1,000 \mathrm{msec}$. After the interpicture interval, pictures appeared for $700 \mathrm{msec}$ simultaneously at both of the $\mathrm{L}$ and $\mathrm{R}$ peripheral locations. The same central stimulus appeared for all four contingency test trials, counterbalanced over infants.

Finally, infants received a set of four disengagement test trials to determine whether they would make a saccade to the peripheral picture when doing so required them to disengage attention from the central stimulus, which remained visible. Disengagement trials began with the presentation of a central stimulus. After $700 \mathrm{msec}$, a peripheral picture (L or R) appeared, along with the central stimulus, for an additional $700 \mathrm{msec}$. Both pictures then disappeared. A 1,000 msec intertrial interval separated successive disengagement test trials. Over the four disengagement trials, each central stimulus appeared twice, in a random order, followed by the L or R pictures. The contingent relation between central stimuli and peripheral targets was preserved during the disengagement test trials for infants who had experienced interevent contingencies during the VExP sequence. The three tasks were completed within $118 \mathrm{sec}$.

\section{Data Reduction}

To facilitate judgments of eye position, the scorer viewed a 1-min sample of the infant's videotape at half normal speed and noted the modal position of the corneal 
reflection on the infant's eye while pictures were displayed on the left, center, and right of the video monitor. These modal positions were used as a frame of reference, individualized for each infant, for judging the direction of the infant's gaze during the detailed coding of eye movements that followed. The scorer then coded the direction and latency of all discernible shifts in visual fixation from the videotaped records. Three classes of eye movements were coded. Anticipations were defined as shifts in fixation that met three criteria: (a) the shift occurred during the interpicture interval or so soon after picture onset (i.e., within $200 \mathrm{msec}$ ) that the eye movement was most likely programmed before picture onset; (b) the shift brought the infant's gaze closer to the upcoming picture than to any other picture location; and (c) the infant's gaze remained at this location at least until the picture appeared. Reactions were defined as shifts that brought the infant's gaze to the location of the current picture and that occurred between 201 and 1,200 msec after picture onset (i.e., within $500 \mathrm{msec}$ of picture offset). Interstimulus interval (ISI) shifts were defined as shifts that occurred during the interpicture interval that did not qualify as anticipations (i.e., because the eye did not move far enough, in the correct direction, or remain long enough at the location of the upcoming picture) or reactions to the picture (i.e., because the eye moved more than $500 \mathrm{msec}$ after picture offset or in the wrong direction). Two experienced scorers independently coded the videotapes of 7 infants to determine interrater reliability. Direction estimates were identical for $96.6 \%$ of all anticipations, $96.3 \%$ of all reactions, and $94.9 \%$ of all ISI shifts. Latency estimates were identical for $84.8 \%$ of anticipations, $85.2 \%$ of reactions, and $73.8 \%$ of ISI shifts, and within one video frame (i.e., $\pm 33.33 \mathrm{msec}$ ) for $93.2 \%$ of anticipations, $95.1 \%$ of reactions, and $86.7 \%$ of ISI shifts.

\section{RESULTS}

\section{Infants' Use of Interevent Contingencies}

To determine whether 3-month-olds use interevent contingencies to form expectations for upcoming events in a paradigm where they have shown sensitivity to spatiotemporal regularity, we examined data from two sources-infants' behavior during the 60-picture VExP sequence and their responses to the four contingency test trials (adapted from Johnson et al., 1991) that followed the VExP task. Infants' sensitivity to spatiotemporal regularity was examined in the same analyses. For the VExP task, we examined three measures: correct anticipation of peripheral pictures, eye movements during the intervals between pictures that did not qualify as anticipations, and saccade latencies. For the contingency test trials that followed the VExP task, we examined the direction of infants' looks to determine if they were consistent with the preceding interevent contingencies or the spatiotemporal pattern of the prior VExP sequence. 
Correct anticipations of peripheral pictures during the VExP. First, we analyzed the percentages of peripheral pictures that infants correctly anticipated in a $2 \times 2$ analysis of variance (ANOVA) with interevent contingency (contingent vs. noncontingent) and spatiotemporal regularity (regular vs. irregular) as factors (see Table 1). A significant main effect for interevent contingency, $F(1,92)=7.74$, $p<.01$, indicated that more peripheral pictures were correctly anticipated during the VExP task when there was a contingent relation between the content of the central picture and the location of the upcoming peripheral picture $(M=26.70 \%$, $S D=9.42)$ than when there was no such contingency $(M=21.21 \%, S D=10.86)$. In addition, a significant main effect for spatiotemporal regularity, $F(1,92)=11.03$, $p<.01$, confirmed that regular sequences were associated with more correct anticipations of peripheral pictures $(M=27.23 \%, S D=10.73)$ than irregular sequences $(M=20.68 \%, S D=9.21)$. These two factors did not interact, $F(1,92)=0.90$, $p>.34$, suggesting that the effect of interevent contingencies did not depend on whether the spatiotemporal sequence was regular or not.

ISI shifts that were not anticipations. ISI shifts did not qualify as anticipations if the eye did not move far enough to reach a peripheral location, if it moved in the wrong direction, or if it did not remain at the correct location until the picture appeared. Analysis of these nonanticipatory eye movements during the ISIs before peripheral pictures confirmed infants' use of interevent contingencies and spatiotemporal regularity. Infants assigned to sequences with interevent contingencies made more shifts toward peripheral locations during the ISIs following central pictures $(M=35.45 \%$ of ISIs, $S D=14.59)$ than did infants assigned to the

TABLE 1

Means and Standard Deviations for Percentage of Anticipations for Peripheral and Central Pictures, and Median Reaction Times for Unanticipated Pictures for Infants in Each Sequence

\begin{tabular}{|c|c|c|c|c|c|c|c|c|}
\hline \multirow[b]{3}{*}{ Sequence } & \multicolumn{4}{|c|}{ Percentage of Anticipations } & \multicolumn{4}{|c|}{ Median RT (in msec) } \\
\hline & \multicolumn{2}{|c|}{$\begin{array}{l}\text { Peripheral } \\
\text { Pictures }\end{array}$} & \multicolumn{2}{|c|}{$\begin{array}{l}\text { Central } \\
\text { Pictures }\end{array}$} & \multicolumn{2}{|c|}{$\begin{array}{l}\text { Peripheral } \\
\text { Pictures }\end{array}$} & \multicolumn{2}{|c|}{$\begin{array}{l}\text { Central } \\
\text { Pictures }\end{array}$} \\
\hline & $M$ & $S D$ & $M$ & $S D$ & $M$ & $S D$ & $M$ & $S D$ \\
\hline $\begin{array}{l}\text { Sequence } 1 \\
\quad \text { (regular }+ \text { contingent) }\end{array}$ & 29.0 & 9.63 & 17.9 & 8.60 & 497 & 140 & 470 & 95 \\
\hline $\begin{array}{l}\text { Sequence } 2 \\
\quad \text { (irregular + contingent) }\end{array}$ & 24.4 & 8.78 & 23.5 & 10.01 & 544 & 145 & 504 & 92 \\
\hline $\begin{array}{l}\text { Sequence } 3 \\
\quad \text { (regular + not contingent) }\end{array}$ & 25.4 & 11.65 & 23.0 & 11.88 & 494 & 90 & 477 & 89 \\
\hline $\begin{array}{l}\text { Sequence } 4 \\
\quad \text { (irregular + not contingent) }\end{array}$ & 17.0 & 8.25 & 24.4 & 10.37 & 532 & 137 & 487 & 88 \\
\hline
\end{tabular}




\section{ISI Shifts}

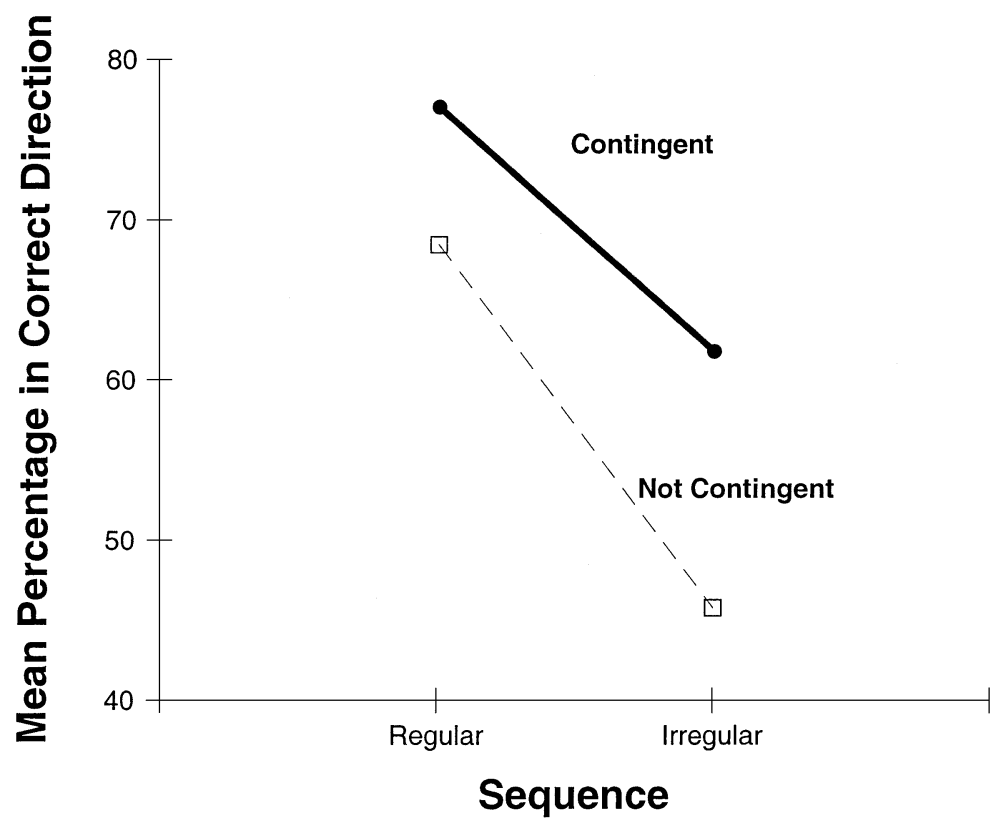

FIGURE 1 Mean percentage of peripheral ISI shifts toward the correct location as a function of spatiotemporal sequence and interevent contingency information.

no-contingency sequences $(M=28.79 \%, S D=15.85), F(1,92)=4.74, p<.05 .^{1}$ Moreover, as Figure 1 shows, peripheral ISI shifts more often headed in the correct direction (i.e., toward the upcoming picture) during contingent $(M=69.26 \%$, $S D=18.47)$ than noncontingent sequences $(M=57.14 \%, S D=24.61), F(1,91)$ $=9.26, p<.01$, and during regular $(M=72.67 \%$ of peripheral shifts, $S D=$ $19.52)$ than irregular sequences $(M=53.80 \%, S D=21.47), F(1,91)^{2}=22.06$, $p<.001$. The contingency and spatiotemporal regularity factors did not interact,

\footnotetext{
${ }^{1}$ In addition to the main effect for contingency, there was also a Contingency $\times$ Spatiotemporal regularity interaction, $F(1,92)=4.86, p<.05$, analysis of which indicated that the contingent sequences were associated with more peripheral ISI shifts regardless of whether the spatiotemporal pattern was regular or not; however, the difference between contingent and noncontingent sequences was significant only for the irregular sequences ( $p<.05$, Bonferroni protected $t$ tests).

${ }^{2}$ Error degrees of freedom have been reduced by one because an infant assigned to Sequence $1 \mathrm{did}$ not make any peripheral ISI shifts.
} 
$F(1,91)=.85, p>.36$. As Figure 1 indicates, infants who saw sequences that lacked both spatiotemporal regularity and interevent contingencies (i.e., Sequence 4 ) made only $45.8 \%$ of their peripheral ISI shifts in the correct direction, a value that did not differ significantly from chance. The percentages of peripheral ISI shifts in the correct direction for the other three groups, in contrast, were all significantly greater than chance $(p<.001)$.

Eye movement latencies during the VExP. Although infants were faster to react to peripheral pictures that were embedded within a regular spatiotemporal sequence $(M=496 \mathrm{msec}, S D=116.36)$ than to those that occurred within an irregular sequence $(M=538 \mathrm{msec}, S D=139.82)$, this difference was not statistically significant, $F(1,92)=2.55, p=.11$. Moreover, interevent contingency information was not significantly related to median reaction time (RT), nor did interevent contingencies interact with spatiotemporal sequence (both $F$ values $<1.0$ ).

Performance during contingency test trials. Infants' responses during the four contingency test trials that followed the VExP sequence provided an additional source of information about whether infants can form expectations based on stable interevent contingencies. Of the 85 infants who responded on the first contingency test trial, only $54 \%$ looked toward the side associated with the central picture. The tendency to look toward the contingent location did not depend on the spatiotemporal regularity properties of the sequence or on the interaction between interevent contingencies and spatiotemporal regularity (all $F$ values $<1.0$ ). On subsequent contingency test trials as well, infants looked at the two peripheral locations about equally often $(M=51.22 \%$ for the contingent side, $S D=35.05)$, regardless of the previous picture sequence. These values are similar to those reported by Johnson et al. (1991) for 2- and 3-month-olds, which were 55.9\% and 55.7\%, respectively.

Although interevent contingencies did not affect the direction of infants' looks during contingency test trials, the preceding spatiotemporal pattern did affect infants' looks. For example, infants assigned to Sequence 1, who watched the regular sequence with stable interevent contingencies, continued the side-to-side alternating pattern during the contingency test trials by looking (for example) left on the first contingency test trial, right on the second, left on the third, and right on the fourth. When anticipations occurred during the contingency test trials, 73\% preserved this alternating spatiotemporal pattern both on the first test trial (i.e., they fixated the side opposite the preceding peripheral picture) and on succeeding test trials. Similarly, $60 \%$ of reactive eye movements (i.e., those that occurred after the two pictures appeared) fixated on the opposite side of either the preceding peripheral stimulus (first test trial) or on successive test trials, in keeping with the alternating pattern of the sequence. Comparable values for infants assigned to Sequence 3 , who saw a regular sequence without interevent contingencies, were $61 \%$ for anticipations that preserved the VExP spatiotemporal pattern and $54 \%$ 
for reactions that preserved the pattern. In contrast, infants who saw the irregular VExP sequence were more likely to revisit the location of the last peripheral picture of the VExP sequence during each contingency test trial. Thus, $74 \%$ of the anticipations during contingency test trials for infants assigned to Sequence 4 (who watched the irregular sequence without interevent contingencies) returned to the side of the last peripheral picture; similarly, $70 \%$ of the reactions following picture onset revisited the location of the last peripheral picture. Comparable values for infants assigned to Sequence 2, who saw an irregular VExP sequence with interevent contingencies, were $63 \%$ for anticipations that revisited the last peripheral location and $58 \%$ for reactions.

To summarize, analysis of anticipations and nonanticipatory ISI shifts during the VExP task indicated that infants used both interevent contingencies and spatiotemporal regularity as sources of information for forming expectations about upcoming events. In contrast, these same infants did not look reliably at the "correct" location during the contingency test trials that followed the VExP task, although there was evidence of infants' sensitivity to spatiotemporal regularity during both the VExP and during the contingency test trials.

\section{Relation Between Anticipation and Disengagement}

A second purpose of the study was to examine the relation between the ability to make anticipatory eye movements and the ability to disengage attention from a visual target. Recall that after the 60-picture VExP sequence and the four contingency test trials, infants received four disengage attention test trials, during which the central stimulus remained visible while a peripheral picture was displayed. If infants experienced difficulty in disengaging attention from a visual target, they should have been less likely to look at the peripheral picture during these disengagement trials, when the central picture remained visible, than during the VExP sequence, when there was a 1,000-msec gap between central and peripheral pictures. Consistent with this hypothesis, infants missed more peripheral pictures during the disengagement test trials $(M=25.8 \%, S D=34.50)$ than during the VExP sequence $(M=6.2 \%$, $S D=6.16)$, a difference that was significant, $F(1,92)=34.55, p<.001$. In addition, median latencies of saccades to peripheral pictures during the disengagement test trials $(M=557 \mathrm{msec}, S D=259.97)$ were longer than latencies to peripheral pictures during the $\operatorname{VExP}(M=517 \mathrm{msec}, S D=129.71)$, although this difference was not significant, $F(1,75)^{3}=2.02, p<.16$. These findings suggest that infants found it more difficult to shift to peripheral pictures while a central picture was visible.

\footnotetext{
${ }^{3}$ Degrees of freedom have been reduced because 17 infants responded on fewer than two disengagement trials, and, as a consequence, median RT values for the disengagement trials were not calculated.
} 
Johnson's (1990) model predicts a developmental sequence in oculomotor control such that infants who are more likely to make anticipatory saccades will also find it easier to disengage from fixated stimuli. To test this prediction, we examined the correlation between two measures of performance on the VExP-percentage of peripheral pictures that were anticipated and median RT for unanticipated peripheral pictures - and two measures of the infant's ability to look away from the central picture during the disengagement test trials - percentage of missed trials and median RT during the disengagement test. Neither measure of VExP performance was significantly related to performance on the disengagement trials (percent VExP anticipation and percent missed disengagement trials, $r=.01$; percent VExP anticipation and median disengagement RT, $r=-.02$; median VExP RT and percent missed disengagement trials, $r=.13$; median VExP RT and median disengagement RT, $r=.10$; all $p$ values $>.20$ ). The two measures of performance during the disengagement trials were themselves correlated $(r=.34, p<.01)$, although the two measures of VExP performance were not $(r=-.04, p>.60)$.

\section{The Role of Infants' Spatial Bias in Performance}

The final purpose of this study was to examine how infants' initial visual search strategies interact with the demands of the modified visual expectation task. In particular, we hypothesized that infants' tendency to make repetitive eye movements during the ISIs between picture presentations would facilitate anticipation of pictures that were consistent with this bias and hinder anticipation otherwise. ${ }^{4}$ To examine this hypothesis, we first analyzed infants' eye movements during segments of the irregular sequences that supported repetitive eye movements (i.e., segments containing $\mathrm{L}, \mathrm{C}, \mathrm{R}$ or $\mathrm{R}, \mathrm{C}, \mathrm{L}$ picture appearances) compared to segments that supported alternating eye movements (i.e., segments containing L, C, L or R, C, R picture appearances). For infants with a bias to make repetitive saccades, anticipation of peripheral pictures should be more likely in the former type of segment than in the latter. This was the case for infants assigned to both of the irregular sequences. Infants who watched Sequence 2 (i.e., irregular + contingent) anticipated significantly more peripheral pictures during the segments that supported repetitive eye movements $(M=28.7 \%, S D=13.31)$ than during the segments that supported alternating eye movements $(M=16.5 \%, S D=8.70), t(23)=3.63, p<.001$, as did infants assigned to Sequence 4 (i.e., irregular + noncontingent), who anticipated

\footnotetext{
${ }^{4}$ Prior to testing this hypothesis, we first confirmed that infants had a bias toward making repetitive shifts during the ISIs between pictures. Repetitive shifts accounted for $67 \%$ of all ISI shifts during the first block of 15 trials, $59 \%$ during the second, $57 \%$ during the third, and $49 \%$ during the fourth. Thus, infants began the session with a bias to respond to picture offset by making an eye movement that repeated the direction of their previous saccade, a tendency that declined over trials.
} 
$19.5 \%(S D=12.00)$ and $13.1 \%(S D=9.78)$ of peripheral pictures in the two types of segments, respectively, $t(23)=6.42, p<.05$.

Similarly, for infants assigned to the regular spatiotemporal sequences, we hypothesized that the tendency to make repetitive eye movements during the ISIs between picture presentations would facilitate anticipation of peripheral pictures and hinder anticipation of central pictures (which required an eye movement in the opposite direction). To test this hypothesis, we subtracted the percentage of anticipations for central pictures from the percentage of anticipations for peripheral pictures and analyzed the resulting difference scores in an ANOVA, with spatiotemporal sequence as a between-subject factor. As predicted, the regular sequences enhanced anticipation of peripheral pictures; infants who received the spatiotemporally regular sequences had a larger difference score $(M=6.76, S D=$ 14.69) than those who received the irregular spatiotemporal sequences $(M=-3.26$, $S D=13.41), F(1,92)=13.16, p<.001$. Moreover, despite the fact that a central picture always followed every peripheral picture for all infants, only infants assigned to Sequence 4, which lacked both spatiotemporal regularity and stable interevent contingency information, anticipated significantly more central pictures than peripheral ones, $t(23)=-3.36, p<.01$.

\section{DISCUSSION}

A primary goal of this study was to determine whether young infants' visual expectations are informed by interevent contingencies as well as by regularity of the underlying spatiotemporal sequence. It was no surprise to find that spatiotemporal regularity enhanced infants' expectations; the informativeness of spatiotemporal regularity for infants as young as 2 months had already been established by several studies using the standard two-location VExP in which infants watched dynamic visual events appear according to simple alternation (i.e., $\mathrm{L}, \mathrm{R}, \mathrm{L}, \mathrm{R} \ldots$ ) or more complicated $2-1(\mathrm{~L}, \mathrm{~L}, \mathrm{R} \ldots$ ) or $2-2(\mathrm{~L}, \mathrm{~L}, \mathrm{R}, \mathrm{R} \ldots$.. ) patterns (see Canfield, Smith, Brezsnyak, \& Snow, 1997, and Haith et al., 1993, for reviews of these studies). The results of this study added to this knowledge base by showing that infants, at least by 3 months, are also able to extract the regularity of a repeating sequence involving three locations. In addition, this study indicated that experience with a regular sequence during the visual expectation task biased infants' looks during a subsequent two-choice contingency test: Infants' looks during contingency test trials continued the side-to-side alternation pattern of the prior VExP sequence. In contrast, infants who experienced an irregular sequence did just the opposite; during the contingency test trials, they looked back to the previously seen location. Consistent with past studies, saccade latencies to unanticipated peripheral pictures were quicker for infants who saw regular sequences than for infants who saw irregular sequences, although this 
difference was not statistically significant. However, the saccade latencies obtained in this study, with means ranging from 470 to $544 \mathrm{msec}$, were commensurate with those of prior visual expectation studies with infants of the same age (means ranging from $373-553 \mathrm{msec}$ ), despite the somewhat greater separation between adjacent pictures $\left(10^{\circ}\right.$ in this study vs. $6^{\circ}$ typical in studies with two locations) and the increased number of locations at which pictures appeared. Thus, the results of this study generally confirmed past findings of infants' remarkable sensitivity to regular spatiotemporal pattern information, despite the use of three, rather than two, picture locations.

The results of this study also revealed infants' remarkable sensitivity to stable interevent contingency information. This finding was quite surprising in light of Johnson et al.'s (1991) earlier report in which they concluded, on the basis of infants' failure to look to the "correct" (cued) side during two-choice contingency test trials, that the contingency learning capability of even 4-month-olds was weak at best. As in the Johnson et al. study, infants' behavior during the contingency test trials in this study also indicated that infants had not learned the contingent relation between the content of the central stimulus and the location of the next peripheral picture: Infants were no more likely to turn toward the cued side than would be expected by chance. However, infants' behavior during the preceding VExP task provided convincing evidence that infants are able to use the stable contingency information to form expectations for upcoming pictures. Specifically, infants anticipated more peripheral pictures when there was a contingent relation between central and peripheral events, and this advantage for contingent sequences was obtained even when the underlying spatiotemporal pattern was irregular. In the absence of stable interevent contingencies, infants tended to shift back to the central location during the intervals between pictures, whereas infants who experienced stable interevent contingencies shifted to peripheral locations and generated ISI shifts that were correct significantly more often than would be expected by chance. Again, this was true even for the sequence that was irregular. Thus, it was clear from their performance in the visual expectation task that these 3-month-old infants were learning from the stable interevent contingencies, even though they did not demonstrate their competence during the two-choice contingency test trials.

How can we explain this discrepancy in findings regarding infants' use of contingency information? Johnson et al. (1991) speculated that anticipatory looks toward cued locations may be a form of implicit learning, whereas turning toward the correct location in a two-alternative forced choice paradigm may require explicit learning. Another possibility is that factors beyond the knowledge of contingent relations may govern infants' choices when faced with two alternatives. Novelty is an obvious candidate (e.g., Fagan \& Haiken-Vasen, 1997), as is experience with recent spatiotemporal patterns. These other factors may interfere with infants' responses that might otherwise express their sensitivity to contingency 
information. A case in point comes from studies of the Moss-Harlow effect (e.g., Valenti, 1985), which nicely illustrates how novelty preferences can interfere with performance on a learning task; young children and individuals with mental retardation consistently score higher on delayed nonmatch to sample tasks than delayed match to sample. Both tasks require the same associative learning and memory capacity but, in the former, participants must choose the novel alternative, whereas in the latter they must choose the familiar. If infants recognize that one side of a two-alternative forced choice test is more novel (or more unusual), this recognition may actually compete with their tendency to look to the "correct" side. In this case, the VExP may provide a more sensitive measure of the infant's sensitivity to contingency information.

A second goal of this research was to examine the relation between the young infant's ability to disengage attention from a visible target and the ability to make voluntary saccades in anticipation of upcoming events. We found no support for the prediction from Johnson's model that there would be a correlation between the ability to disengage attention and the frequency of anticipatory saccades. Consistent with prior studies (e.g., Atkinson et al., 1988; Atkinson et al., 1992; Frick et al., 1999; Hood \& Atkinson, 1993; Johnson et al., 1991), we found that the presence of a central stimulus interfered with infants' saccades to a peripheral target. However, the magnitude of the interference that the visible central stimulus produced was much smaller than we expected from past research. For example, in the Johnson et al. (1991) study, 3-month-old infants looked at the peripheral picture on only $45 \%$ of the disengagement test trials compared to $75 \%$ of the test trials in this study. Similarly, most of the peripheral saccades that 3-month-olds generated during disengagement tests in the Johnson et al. (1991) study had latencies over $500 \mathrm{msec}$; many (47\%) had latencies over 1000 msec. Hood and Atkinson (1993) and Frick et al. (1999) also reported dramatically longer saccade latencies $(M=1.8 \mathrm{sec}$ and $1.16 \mathrm{sec}$ ) for 3-month-olds when a central stimulus remained visible while a peripheral target was displayed (i.e., in their overlap conditions). By comparison, mean saccade latency to peripheral pictures during disengagement trials in this study was $557 \mathrm{msec}$, more than three times faster than the value reported by Hood and Atkinson (1993), and more than two times faster than the value reported by Frick et al. (1999). This is despite the fact that mean latencies among the three studies were very similar (within $58 \mathrm{msec}$ ) when we compared the 720-msec gap condition of the Hood and Atkinson (1993), and the 750-msec gap condition of the Frick et al. (1999) study, to the 1,000-msec gap that we used in the visual expectation task. The lower latency and higher rate of successful looks away from the central target during the disengagement task of this study suggest that our measure of disengagement may have been less sensitive than comparable measures of prior studies. Thus, further tests of the predicted relation between the ability to disengage from a central target and the frequency of anticipatory saccades may succeed with a more sensitive measure of the ability to disengage visual fixation. 
Several factors may explain why infants were better able to disengage from the central stimulus during the disengagement trials of this study. Peripheral pictures in this study were only $10^{\circ}$ from the center of the monitor, compared to $23^{\circ}, 25^{\circ}$, and $34^{\circ}$ in the Hood and Atkinson (1993), Frick et al. (1999), and Johnson et al. (1991) studies, respectively. Previous studies have shown that retinal eccentricity affects the relative salience of peripheral targets both for infants (e.g., Aslin \& Salapatek, 1975) and adults (e.g., Findlay, 1980; Newby \& Rock, 1998). Thus, the smaller visual angle separating the central and peripheral pictures in this study may have made the peripheral pictures more salient, in turn making it easier for infants to disengage from the central picture. Also, infants had extensive practice at shifting from the center to the side during the 60-picture visual expectation task that preceded the disengagement test trials. It is possible that the expectations infants built up over the course of the visual expectation task enabled them to disengage attention more easily from the central picture than might otherwise be the case. Regardless of its source, infants' relative facility in disengaging from a central stimulus suggests that, even among young infants, the ability to disengage attention depends on situational and experiential factors in addition to the maturational state of the underlying neurological mechanisms. Of course, this study does not address whether retinal eccentricity or experience in the visual expectation task were key factors in mediating this effect, or whether infants younger than 3 months would also experience a reduction in the power of the central stimulus to capture attention. Further studies are needed to identify the situational and experiential factors that can affect the ease with which infants engage and disengage visual attention and the ages at which these factors operate.

The third goal of this study was to examine how infants' initial search strategies interact with performance on the visual expectation task. This study confirmed an earlier finding (Wentworth \& Haith, 1998) that 3-month-old infants began the session with a bias to repeat the direction of their last (successful) saccade when the picture under scrutiny disappears. In a two-location alternation task, this bias must be overcome if infants are to anticipate upcoming pictures. In this three-location task, this bias supported anticipation of peripheral pictures but interfered with anticipation of central pictures. As predicted, infants who received the regular alternation sequences anticipated more peripheral pictures than central, a finding that is consistent with infants' purported bias to make repetitive eye movements during the interpicture intervals of the visual expectation task. In fact, only infants who watched the sequence that lacked both spatiotemporal regularity and stable interevent contingencies anticipated more central than peripheral pictures despite the fact that central pictures were completely predictable_-after every peripheral picture a central picture always followed.

The relative difficulty infants had in anticipating the central picture, despite its high predictability, suggests that infants' bias to make repetitive saccades was 
fairly persistent. It is also possible that infants could have more readily supplanted this bias with an alternative search strategy, such as looking in the opposite direction, if the sequence had given them more consistent information that their preferred strategy was no longer appropriate. Although the two-location alternating sequence delivers this type of reliable information - every time a new picture appears, it is always in the opposite location-the three-location alternation sequence does not. In the regular three-location sequence, and in the irregular sequences with reliable interevent contingency information, infants' initial repetitive strategy would pay off by yielding a look in the correct direction about half of the time (i.e., for all peripheral targets). Only in the irregular sequence without interevent contingency information would the infants' repetitive search strategy fail more than half of the time. Yet despite the inconsistent information that infants received from the three-location sequence, they were able to modify their initial repetitive search strategy, and they did so fairly rapidly. The percentage of repetitive interpicture shifts declined from $67 \%$ in the first block of 15 trials to $59 \%$ in the second. It is possible that, with additional experience, infants would have eventually been able to anticipate peripheral and central pictures with equal facility.

The results of this study suggest that the VExP can be easily modified to address these and other questions. The three-location version of the VExP permits a wide range of possible spatiotemporal patterns as well as inclusion of various types of interevent contingencies. Moreover, two recent studies (Csibra, Johnson, \& Tucker, 2001; Wentworth, Haith, \& Karrer, 2001) have shown that it may be fruitful to combine measures of the brain's cortical electrical potentials with measures of infants' visual behavior in the VExP. Future studies of infants' anticipations, reactions, and ISI shifts in the modified VExP, coupled with appropriate brain measures, should shed additional light on the development of visual expectations early in life, as well as on the development of the oculomotor systems. In particular, it should be possible to examine the respective roles that parietal and frontal areas play as infants process central cues and engage and disengage attention from expected and unexpected peripheral targets (e.g., Richards, 2001).

Finally, we would be remiss if we did not mention a few caveats about the process of relating brain development to mastery of general skills and to age differences in performance on the specific tasks that we present to infants. As Haith (1998) noted, there is a tendency to view the early acquisition of various capabilities as an all-or-none discrete process, and the maturation of brain function as if a switch were turned on in the head. For example, we may assume that infants will make anticipatory eye movements, voluntarily disengage attention from a target on the fovea, and learn interevent contingencies when the brain is mature enough, but not before. However, there is a good deal of evidence that argues against this simplistic view. For example, Smith and Canfield (1999; Smith, 1999) showed that 2-month-old infants used one type of contingency 
information, motion of a central cue, to make predictive saccades to the locations of peripheral targets, but they did not use another type of contingency, between the cue's color and the location of the next target, in the same paradigm. Thus, the ability to learn a visual contingency and to use this information to make predictive saccades depended on the type of visual information (motion vs. color) contained in the cue. In this study, infants used interevent contingencies to make anticipatory saccades and visual shifts during interpicture intervals of the visual expectation task, but they did not use these same contingencies to look preferentially to the "correct" location during two-choice contingency test trials. Thus, infants' capacity to use contingency information was revealed in one task but not another. Moreover, infants readily disengaged visual attention from a central picture in this study but not in previous studies (e.g., Hood \& Atkinson, 1993; Johnson et al., 1991) with larger retinal eccentricities and different trial sequences. These discrepancies convince us of the need, as we attempt to make inferences about brain-behavior correspondences, to study infants' developing capabilities in a wider variety of tasks, with systematic variation of important parameters, supplemented by brain imaging techniques, where possible.

\section{ACKNOWLEDGMENTS}

This research was supported by U.S. Public Health Service Postdoctoral Fellowship MH09298, National Institute of Child Health and Human Development Research Grant HD20026, and National Institute of Mental Health Research Scientist Award MH00367. We thank the infants and parents who participated.

A preliminary report of the data presented here was presented at the 1995 biennial meeting of the Society for Research in Child Development in Indianapolis, IN.

\section{REFERENCES}

Aslin, R. N., \& Salapatek, P. (1975). Saccadic localization of visual targets by the very young human infant. Perception and Psychophysics, 17, 293-302.

Atkinson, J., Hood, B., Braddick, O. J., \& Wattam-Bell, J. (1988). Infants' control of fixation shifts with single and competing targets: Mechanisms for shifting attention. Perception, 17, 367-368.

Atkinson, J., Hood, B., Wattam-Bell, J., \& Braddick, O. J. (1992). Changes in infants' ability to switch visual attention in the first three months of life. Perception, 21, 643-653.

Canfield, R. L., Smith, E. G., Brezsnyak, M. P., \& Snow, K. L. (1997). Information processing through the first year of life: A longitudinal study using the visual expectation paradigm. Monographs of the Society for Research in Child Development, 62(2, Serial No. 250).

Csibra, G., Johnson, M., \& Tucker, L. (2001). Differential frontal cortex activation before anticipatory and reactive saccades in infants. Infancy, 2, 159-174. 
Fagan, J. F., \& Haiken-Vasen, J. (1997). Selective attention to novelty as a measure of information processing across the lifespan. In J. A. Burack \& J. T. Enns (Eds.), Attention, development, and psychopathology (pp. 55-73). New York: Guilford.

Findlay, J. M. (1980). The visual stimulus for saccadic eye movements in human observers. Perception, 9, 7-21.

Fisher, B., \& Breitmeyer, B. (1987). Mechanisms of visual attention revealed by saccadic eye movements. Neuropsychologica, 25, 73-83.

Frick, J. E., Colombo, J., \& Saxon, T. F. (1999). Individual and developmental differences in disengagement of fixation in early infancy. Child Development, 70, 537-548.

Haith, M. M. (1998). Who put the cog in infant cognition? Is rich interpretation too costly? Infant Behavior and Development, 21, 167-179.

Haith, M. M., Hazan, C., \& Goodman, G. S. (1988). Expectation and anticipation of dynamic visual events by 3.5-month-old babies. Child Development, 59, 467-479.

Haith, M. M., Wentworth, N., \& Canfield, R. L. (1993). The formation of expectations in early infancy. In C. Rovee-Collier \& L. P. Lipsitt (Eds.), Advances in infancy research (Vol. 8, pp. 251-297). Norwood, NJ: Ablex.

Hood, B. M. (1995). Shifts of visual attention in the human infant: A neuroscientific approach. In L. Lipsett \& C. Rovee-Collier (Eds.), Advances in infancy research (Vol. 9, pp. 163-216). Norwood, NJ: Ablex.

Hood, B. M., \& Atkinson, J. (1993). Disengaging visual attention in the infant and adult. Infant Behavior and Development, 16, 405-422.

Johnson, M. H. (1990). Cortical maturation and the development of visual attention in early infancy. Journal of Cognitive Neuroscience, 2, 81-95.

Johnson, M. H., Posner, M. I., \& Rothbart, M. K. (1991). Components of visual orienting in early infancy: Contingency learning, anticipatory looking, and disengaging. Journal of Cognitive Neuroscience, 3, 335-344.

Kaas, J. H. (1989). Why does the brain have so many visual areas? Journal of Cognitive Neuroscience, $1,121-135$.

Newby, E. A., \& Rock, I. (1998). Inattentional blindness as a function of proximity to the focus of attention. Perception, 27, 1025-1040.

Reznick, J. S. (1994). In search of infant expectation. In M. M. Haith, J. B. Benson, \& R. J. Roberts, Jr. (Eds.), The development of future-oriented processes (pp. 39-59). Chicago: University of Chicago Press.

Richards, J. E. (2001). Cortical indexes of saccade planning following covert orienting in 20-week-old infants. Infancy, 2, 135-157.

Smith, E. G. (1999, April). Early indication of prefrontal cortex function: Visual expectations in two-month-old infants. Poster session presented at the biennial meeting of the Society for Research in Child Development, Albuquerque, NM.

Smith, E. G., \& Canfield, R. L. (1999). Predictive saccadic eye movements in 2-month-old infants: Early frontal lobe function within the dorsal visual stream. Unpublished manuscript.

Valenti, S. S. (1985). Children's preference for novelty in selective learning: Developmental stability of change? Journal of Experimental Child Psychology, 40, 406-419.

Wentworth, N., \& Haith, M. M. (1992). Event-specific expectations of 2- and 3-month-old infants. Developmental Psychology, 28, 842-850.

Wentworth, N., \& Haith, M. M. (1998). Infants' acquisition of spatiotemporal expectations. Developmental Psychology, 34, 247-257.

Wentworth, N., Haith, M. M., \& Karrer, R. (2001). Behavioral and cortical measures of infants' visual expectations. Infancy, 2, 175-195.

Zeki, S. (1993). A vision of the brain. Oxford, England: Blackwell. 